\title{
QiShenYiQi Dripping Pill Alleviates Myocardial Ischemia-Induced Ferroptosis Via Improving Mitochondrial Dynamical Homeostasis and Biogenesis
}

\section{Lingling Wu}

China Pharmaceutical University

\section{Zhaoyang Fan}

China Pharmaceutical University

Jincheng Liu

China Pharmaceutical University

\section{Zekun Cui}

China Pharmaceutical University

\section{Zixuan Huang}

China Pharmaceutical University

Qingfei Zhao

China Pharmaceutical University

\section{Yuanyuan Zhang}

China Pharmaceutical University

\section{Boyang Yu}

China Pharmaceutical University

\section{Junping Kou}

China Pharmaceutical University

\section{Fang Li ( $\nabla$ lifangcpu@163.com )}

China Pharmaceutical University https://orcid.org/0000-0002-7617-6534

\section{Research Article}

Keywords: QiShenYiQi dripping pill, myocardial ischemia, ferroptosis, mitochondrial biogenesis, mitochondrial dynamics

Posted Date: March 3rd, 2022

DOI: https://doi.org/10.21203/rs.3.rs-1400480/v1 
License: (c) (i) This work is licensed under a Creative Commons Attribution 4.0 International License. Read Full License 


\section{Abstract}

\section{Purpose}

QiShenYiQi dripping pill (QSYQ) is a traditional Chinese medicine for alleviating cardiovascular diseases. Whereas, the potential mechanism remains to be further elucidated. Thus, this paper is designed to explore the protective mechanism of QSYQ in myocardial ischemia (MI)-induced injury.

\section{Methods}

Myocardial ischemia was induced in mice by left anterior descending coronary artery ligation (CAL). Then, mice were treated by QSYQ dripping pills $(12,35$ and $105 \mathrm{mg} / \mathrm{kg}$ ) for 14 days. The effect and mechanism of QSYQ against MI injury were investigated in vivo.

\section{Results}

Treatment with QSYQ significantly improved the contractile function, and extenuated myocardial fibrosis and inflammatory cell infiltration after MI injury. QSYQ administration also reduced the level of LDH and NT-pro BNP in serum. Additionally, myocardial iron content and serum MDA, 4-HNE were decreased and GSH was increased after QSYQ administration. The mRNA expression of GPX4 and SLC7A11 were increased, and PTGS2 mRNA expression level was reduced by QSYQ treatment. Moreover, QSYQ ameliorated myocardial mitochondrial ultrastructure damage and decreased mitochondrial ROS production. Furthermore, QSYQ heightened the expressions of PGC-1a, TFAM and Nrf1, which indicated that QSYQ could promote mitochondrial biogenesis. In addition, QSYQ treatment also increased MFN2 and OPA1 expressions, while the expression of p-DRP1 was decreased.

\section{Conclusions}

All these suggested that QSYQ could promote mitochondrial dynamic homeostasis to improve myocardial injury. QSYQ could alleviate Ml-induced ferroptosis via improving mitochondrial dynamical homeostasis and biogenesis, which provided references for its clinical application.

\section{Introduction}

The world's biggest killer is ischemic heart disease, responsible for $16 \%$ of the world's total deaths according to the World Health Organization report in 2019. Severe ischemic events result in massive loss of cardiomyocytes, which separates the infarcted area from the rest of the myocardium, ultimately leading to loss of contractile function, pathological remodeling of the heart, and ultimately heart failure (HF)[1]. HF remains a global pandemic that represents a leading cause of morbidity and mortality worldwide, which seriously threatens the health and quality of human life [2]. And HF still represents a major social and economic burden. HF with reduced ejection fraction is associated with pathological structural, cellular, and molecular changes of the heart, leading to impaired cardiac function. Given the high risk of HF, targeted prevention remains an urgent need. 
Recently, ferroptosis has been found to play a critical role in HF. Ferroptosis is a form of regulated cell death that is characterized by iron overload, leading to accumulation of lethal levels of lipid hydroperoxides [3]. Iron metabolism and activity of GPX4 are thus two major pathways that regulate sensitivity to ferroptosis and have relations with lipid peroxidation [4]. And lipid peroxide level is elevated in myocardia of patients with HF [5]. Therefore, inhibition of ferroptosis is an important therapeutic target for improving HF.

Mitochondria provides primary source of energy for organs and tissues, which is essential for the contractile function of the heart [6, 7]. Dysregulation of mitochondria emerges as a common pathology for $\mathrm{HF}$ and involved in the early pathophysiological stage of $\mathrm{HF}$, which is increasingly recognized as a significant factor in the progression of HF. Normal mitochondrial morphology needs the balanced fusion and fission, which can meet metabolic demands and removal of damaged organelles [8,9]. Research shows imbalanced fusion and fission events disrupt mitochondrial homeostasis and normal heart activity, which are regulated by the Dynamin-related protein 1 (Drp1), mitofusin 2 (MFN2), and optic atrophy 1 (OPA1) [10]. Additionally, mitochondrial biogenesis mediated by peroxisome proliferatoractivated receptor gamma coactivator $1 \mathrm{a}$ (PGC-1a) [11] regulates the mass, distribution and activity of mitochondria and maintains the stability of intracellular environment [9]. Moreover, mitochondrial abnormality also refers to the increased production of reactive oxygen species that is closely associated with ferroptosis [12]. Thus, promoting mitochondrial biosynthesis and mitochondrial homeostasis is an important way of clinical treatment for heart failure.

QiShenYiQi dripping pill (QSYQ) is a traditional Chinese medicine composed of Astragalus membranaceus (AM, Huangqi), Salvia miltiorrhiza (SM, Danshen), Panax notoginseng (PN, Sanqi), and Dalbergia odorifera (DO, Jiangxiang). Previous research has shown that QSYQ could be used in clinical treatment of various heart diseases with beneficial effects on blood circulation and pain relief [13]. Moreover, modern pharmacological researches have shown that QSYQ could ameliorate ischemic heart failure [14], hypertensive nephropathy [15], and ischemia-reperfusion injury [16]. It was also reported that QSYQ could increase the expression of VEGF and promote cardiac angiogenesis [17]. Additionally, it could reduce the calcium overload, prevent the mitochondrial permeability transition pore (MPTP) opening and decrease oxidative stress damage [18]. QSYQ also increased ATP production and ATP synthase activity [19]. However, whether QSYQ could affect mitochondrial dynamics and biogenesis to mitigate myocardial ischemia-induced ferroptosis remains to be further explored. Therefore, this study was designed to elucidate the effect and mechanism of QSYQ on myocardial ischemia-induced ferroptosis and enrich the clinical application basis of QSYQ.

\section{Materials And Methods}

\section{Drugs and Reagents}

QSYQ were provided by Tasly Pharmaceutical Co., Ltd. (Tianjin, China) with the production batch number of 200402. The biochemical kits for detection of lactate dehydrogenase (LDH), malondialdehyde (MDA), 
glutathione (GSH) and tissue iron determination were obtained from Nanjing Jian Cheng Biotechnology Company (Jiangsu, China). The ELISA kits for detection of precursor brain natriuretic peptide (NT-pro BNP) and 4-hydroxynonenal (4-HNE) were obtained from Shanghai Heng Yuan Biotechnology Company (Shanghai, China). RIPA lysis buffer, protease inhibitor, and enhanced chemiluminescence (ECL) reagent were purchased from Vazyme Biotech Co., Ltd. (Jiangsu, China). Hoechst33342 staining kit was provided by Beyotime (Nanjing, China). Antibody against $\beta$-actin were provided by Proteintech Group (IL, USA) and antibody against PGC-1a, TFAM, Nrf1 were purchased from Santa Cruz (CA, USA). Antibody against FHC, GPX4, DRP1, phospho-DRP1, MFN-2 and OPA1 were obtained from Proteintech Group (IL, USA) and antibody against ACSL4 were obtained from Abclonal Proteinch (Wuhan, China).

\section{Animal Experiments}

ICR male mice (20-25 g) were purchased from the Comparative Medical Center of Yangzhou University (Jiangsu, China). Experimental animal production license number: SYXK 2021-0011. The animals were maintained on food and water in standard cages, at controlled temperature $\left(22 \pm 2^{\circ} \mathrm{C}\right)$, relative humidity (40\%-80\%), and $12 \mathrm{~h}$ light/dark cycles.

\section{Animals And Cal Model}

Mice were anesthetized with intraperitoneal injection of pentobarbital. Then, the thoracotomy was operated at the left third and fourth ribs intercostal space, and the heart was exposed followed by making a slipknot (6-0 silk) around the left anterior descending coronary. After ligation, the heart was promptly placed back in the intrathoracic cavity with the expulsion of air.

The surviving mice were randomly divided into six groups according to the experimental requirements: the sham group, the model group (CAL 14 days), the QSYQ low-dose group ( $12 \mathrm{mg} / \mathrm{kg}$, ig), the QSYQ medium-dose group (35 mg/kg, ig), the QSYQ high-dose group (105 mg/kg, ig) and the Enalapril (EP) group $(2.6 \mathrm{mg} / \mathrm{kg}$, ig). The sham and model groups received an equal volume of physiological saline intraperitoneally. Mice in the administration group were treated for 14 days according to the experimental protocol.

\section{Echocardiography}

Mice were anesthetized with $2 \%$ isoflurane and then subjected to transthoracic echocardiography with the Vevo3100 imaging system to assess cardiac structure and function. Body temperatures were maintained between $36.9^{\circ} \mathrm{C}$ and $37.3^{\circ} \mathrm{C}$. The heart rates were maintained at $400-550 \mathrm{bpm}$. At the target heart rate, the position and direction of ultrasonic probe were adjusted to find the maximum of the opening and closing apical value, and the left ventricular echocardiography was obtained. M-mode images were used to assess parameters of left ventricular function. 


\section{Histopathologic Examination}

Mice were sacrificed, the hearts were harvested and fixed in 4\% paraformaldehyde solution for at least 24 $\mathrm{h}$ at $4{ }^{\circ} \mathrm{C}$. The samples were dehydrated and embedded in paraffin for slicing, the thick sections $(4-5 \mu \mathrm{m})$ were stained with hematoxylin-eosin (HE) and Masson according to the manufacturer's instructions (Servicebio Company, Wuhan, China). Then, histopathological changes were examined via an optical microscope (DX45 microscope; Olympus), quantification was performed by Image $\mathrm{J}$ software.

\section{Serum Biochemical Indicators Detection}

After echocardiography measurements, blood samples were collected by extracting the eyeball, rested for $60 \mathrm{~min}$ at room temperature then centrifuged at $3500 \mathrm{rpm}$ for $10 \mathrm{~min}$ at $4^{\circ} \mathrm{C}$, and separated to get serum. The levels of LDH, NT-pro BNP, MDA, 4-HNE and GSH were measured with kits according to the manufacturer's protocols.

\section{Transmission Electron Microscopy}

To observe the cardiac mitochondrial morphology, the fresh heart was removed rapidly fixed with $4 \%$ paraformaldehyde solution including 2.5\% glutaraldehyde for $24 \mathrm{~h}$, and cut into $1 \mathrm{~mm} 3$ pieces (Leica, EMUC7, Germany). The ultrastructure was detected by using transmission electron microscopy (FEI Tecnai G220 TWIV, United States). All the samples were examined under the transmissive electron microscope. Mitochondrial lengths, mitochondrial numbers and mitochondrial volume was performed using Image J software.

\section{Immunofluorescence Analysis}

The fresh heart tissues removed from mice each group were collected and immediately cut into $5 \mu \mathrm{m}$ thick frozen sections. The cardiac tissues were incubated with Mito-SOX Red dye in dark humidified chamber at $37^{\circ} \mathrm{C}$ for $30 \mathrm{~min}$. Then the tissues were stained with Hoechst 33342 for $5 \mathrm{~min}$ in the dark. The images were acquired by a confocal laser scanning microscope (LSM700, Zeiss, Jena, Germany).

\section{Quantitative Real-time Pcr}

Total RNA was isolated from the heart tissues via using Trizol lysis buffer (Vazyme Biotech Co., Ltd, China). RNA concentration was measured by Nanodrop from Thermo Fisher Scientific (San Jose, CA, USA). Following the manufacture instructions, reverse transcription of RNA was performed with the cDNA Reverse Transcription kit (Vazyme Biotech Co., Ltd, China). The mRNA expression of prostaglandinendoperoxide synthase 2(PTGS2), solute carrier family 7 member 11(SLC7A11) and glutathione 
peroxidase 4(GPX4) were measured by real-time fluorescence quantitative PCR. The sequences of the primer pairs are as Tabel1.

\section{Western Blotting}

Heart tissues were lysed in RIPA lysis buffer (Beyotime, Jiangsu, China) with $1 \mathrm{mM}$ PMSF. The lysates were centrifuged at $12000 \times \mathrm{g}$ for $10 \mathrm{~min}$ at $4^{\circ} \mathrm{C}$ and the supernatants were collected. The protein concentrations were measured utilizing a BCA protein assay kit (Beyotime, P0011). The same amount of protein $(30 \mu \mathrm{g})$ was separated by SDS-PAGE and transferred to PVDF membranes (Millipore, Bedford, MA, USA). After blocked with 5\% BSA for $2 \mathrm{~h}$ at room temperature, the membranes were incubated with indicated primary antibody overnight at $4^{\circ} \mathrm{C}$. The primary antibodies against PGC-1a, TFAM, Nrf1, DRP1, p-DRP1, Mfn-2, OPA1, FHC, ACSL4, GPX4, and $\beta$-actin were mixed with the corresponding dilutions $(1: 1000,1: 1000,1: 1000,1: 2000,1: 2000,1: 2000,1: 2000,1: 2000,1: 2000,1: 8000)$. The membrane was washed and incubated with secondary antibody. Later, the membrane was submerged in an ECL reagent developing solution and the data were quantified using Image Lab ${ }^{\mathrm{TM}}$ Software (version 4.1; Bio-Rad).

\section{Statistical Analysis}

All statistical analyses were analyzed using GraphPad Prism version 8.0. All values in the figures were expressed as mean $\pm S D$. Comparisons were performed with one-way analysis of variance (ANOVA). Statistical significance was considered to be present at $p<0.05$.

\section{Results}

\section{QSYQ effectively restored the cardiac function in CAL- induced HF mice}

We used echocardiography to assess the changes of cardiac function 14 days after CAL in the mice. As shown in Fig. 1, when compared with the sham group, echocardiography assessment indicated that CALinduced attenuation of left ventricular contractile function, evidenced by a significant reduction in LVEF, LVEF and stroke volume. Then, mice were injected with QSYQ at the dose of 12,35 or $105 \mathrm{mg} / \mathrm{kg}$ daily, these were markedly reversed by QSYQ in CAL-treated mice (Fig. 1b-d). Moreover, QSYQ could significantly decreased in LV vol (Fig. 1e). Enalapril had shown the same effect as QSYQ. Overall, these results suggested that QSYQ treatment protected the CAL mice from LV remodeling and cardiac dysfunction.

\section{Qsyq Attenuated Myocardial Injury In Cal-induced Hf Mice}

As demonstrated in Fig. 2(a-d), HE staining showed that there was myocardial injury observed in CALinduced HF mice, and a number of inflammatory cells infiltrated into the myocardial tissue. Masson's 
trichrome staining showed that nuclei were absent, and fibroblasts had infiltrated into the heart of model group. Whereas, QSYQ (35 mg/kg, $105 \mathrm{mg} / \mathrm{kg})$ and Enalapril treatment alleviated the histopathological damage in the heart and reduced the fibrosis. Furthermore, compared with the increased serum levels of LDH and NT-pro BNP in CAL-induced HF mice, all three doses of QSYQ reduced the LDH content, and NTpro BNP serum level was decreased only at high dose of QSYQ (Fig. 2e, f). And Enalapril treatment reduced levels of both.

\section{Qsyq Mitigated Ferroptosis In Cal-induced Hf Mice}

As illustrated in Fig. 3(a-c), the serum levels of MDA, 4-HNE were significantly increased and GSH serum level was decreased in CAL-induced HF mice. Moreover, the amount of total iron in heart tissue was elevated after CAL-induced injury (Fig. 3d). MDA, 4-HNE serum levels were markedly reduced by QSYQ administration. And GSH level was increased after QSYQ (35 mg/kg, $105 \mathrm{mg} / \mathrm{kg})$ treatment. In addition, PCR results indicated that the mRNA expression of GPX4 and SLC7A11 were decreased, while PTGS2 mRNA expression level was increased in CAL-induced myocardial injury. After QSYQ treatment, the mRNA expression of GPX4 and SLC7A11 were increased (Fig. 3e-g). PTGS2 mRNA expression level was reduced by QSYQ (35 mg/kg, $105 \mathrm{mg} / \mathrm{kg})$ treatment. Compared with sham group, the expressions of Acyl-CoA Synthetase Long Chain Family Member 4(ACLS4) and Ferritin Heavy Chain(FHC) increased, and GPX4 protein expression was decreased in model group. However, the expression of ACLS4 was decreased and GPX4 expression was increased by QSYQ treatment, while the expression of FHC was reduced by QSYQ $(105 \mathrm{mg} / \mathrm{kg}$ ) administration (Fig. 3h, i). These data revealed that QSYQ could reduce the lipid peroxidation level to alleviate myocardia ferroptosis.

\section{Qsyq Improved The Mitochondrial Ultrastructure And Decreased Mitochondrial Ros Production In Cal-induced Hf Mice}

TEM was used to observe mitochondrial ultrastructure in CAL-induced HF mice. In 2-week CAL hearts, alterations in the shape, numbers and size of mitochondria were evident (Fig. 4a). Quantitative analysis showed in (Fig. 4b, c), the mean number of mitochondria and the ratio of major and minor axes were decreased. After mice treated with QSYQ (105 mg/kg), myocardial morphology was improved, the mean number of mitochondria and the ratio of major and minor axes were increased. All above suggested that QSYQ could improve mitochondrial ultrastructure damage in CAL-induced HF mice. Then, as showcased in Fig. 5(a, b), representative images of the fluorescence, mitochondrial ROS production in the model group was significantly higher than that in the sham group. Whereas, fewer ROS were observed in myocardial tissue with QSYQ treatment. The immunofluorescence result indicated that QSYQ treatment significantly attenuated CAL-induced mitochondrial ROS generation in CAL-induced HF mice. 


\section{Qsyq Promoted Mitochondrial Biogenesis And Mitochondrial Dynamical Homeostasis In Cal-induced Hf Mice}

As shown in Fig. 6, western blotting showed a significant decrease in the expressions of PGC-1a, TFAM and Nrf1, indicating that the CAL-induced cardiac injury was suppressed mitochondrial biogenesis. QSYQ (105 mg/kg) treatment markedly increased PGC-1a, TFAM and Nrf1 expression. As shown in Fig. 7a, DRP1 phosphorylation at Ser616 was increased in the model group, while the MFN2 and OPA1 expressions were both decreased by CAL injury. After the QSYQ (35 mg/kg, $105 \mathrm{mg} / \mathrm{kg})$ treatment, the pDRP1 expression was decreased. And the expressions of OPA1 and MFN-2 were increased by QSYQ (105 $\mathrm{mg} / \mathrm{kg}$ ) treatment (Fig. 7b, c). These results indicated that QSYQ could improve the balance of mitochondrial fusion and fission in CAL-induced HF mice.

\section{Discussion}

Previous researches have shown that QSYQ has the benefits to cardiovascular diseases. QSYQ can be used to improve ventricular remodeling in patients with chronic heart failure, and it can also improve heart failure with preserved ejection fraction $[20,21]$. Pharmacological studies have shown that QSYQ can improve ischemia-reperfusion injury and cardiac hypertrophy by regulating mitochondrial energy metabolism and oxidative stress [18, 19,22]. Moreover, major ingredients in QSYQ have effects on myocardial microcirculatory disturbance, cardiac injury and fibrosis induced by ischemia-reperfusion. Dihydroxylphenyl lactic acid (DLA) from SM could improve energy metabolism and inhibit myocardial cell apoptosis. In addition, astragaloside IV (ASIV), ginsenoside Rb3, and DLA have an anti-inflammatory effect by acting at TLR4 and NF-KB pathways, which are the ingredients from AM, PN and SM. [16]. However, the effect of QSYQ on mitochondrial biogenesis and dynamical balance is not clear. In this work, we not only confirmed the pharmacological effect of QSYQ on improving cardiac function and myocardial damage in CAL-induced HF, but also elucidated that QSYQ could promote mitochondrial biogenesis and dynamical homeostasis to alleviate cardiac ferroptosis.

A vital pathological factor in the development of HF is the death of end-stage cardiomyocytes [23]. Many findings suggest ferroptosis is a potential therapeutic target for various heart disease [24]. Ferroptosis is an emerging form of regulated cell death with iron- and ROS-dependent [25]. The morphological features of ferroptosis are mainly damage to mitochondrial structure such as fragmentation, mitochondrial swelling, and loss of mitochondrial cristae, which is also the pathological process of HF. Mitochondrial ultrastructural damage is often accompanied by increased production of reactive oxygen species, which are thought to be the main pathogenic mechanism and play an important role in cardiac remodeling of $\operatorname{HF}[7,26,27]$. The other characteristics of ferroptosis are the activating lipoxygenases and the decreased GPX4 activity [28]. GPX4 is an antioxidant enzyme, and overexpression of GPX4 improves myocardial injury [29]. The activation of lipid peroxides and the loss of GPX4 activity can lead to oxidative stress, and the inactivation of GPX4 is associated with the production of mitochondrial ROS [30], which partially 
indicates that mitochondrial dysfunction is closely related to ferroptosis. QSYQ is a traditional Chinese medicine composition, which has been reported to improve oxidative stress damage and apoptosis in HF $[8,31]$. However, whether QSYQ can alleviate myocardial injury by inhibiting the production of mitochondrial ROS and ferroptosis has not been reported. In present study, we demonstrated that the expression of ACLS4 and FHC were decreased, and the protein expression of GPX4 was elevated after QSYQ administration. Additionally, the mRNA expressions of SLC7A11 and PGTS2 increased after CAL injury, but decreased after QSYQ administration. Then, transmission electron microscopy was performed to observe mitochondrial ultrastructure. In our research, we observed that mitochondria were swollen, cristae disappeared, and the number of mitochondria was decreased, the ratio of long axis and short axis of mitochondria were decreased in CAL-induced HF, but the changes of mitochondrial damage were improved after QSYQ administration. Moreover, we detected the ROS content of myocardial mitochondria. After CAL injury, ROS production in myocardial mitochondria was significantly increased. However, the content of ROS in myocardial mitochondria was significantly decreased after administration of QSYQ. These data partially illustrated that QSYQ could attenuate mitochondrial ROS and cardiac ferroptosis to alleviate CAL-induced myocardial injury.

Mitochondria are energy organelles that provide basic energy for various life activities and significant in maintaining cardiac homeostasis [9]. Mitochondria affect processes such as energy metabolism, oxidative stress, and apoptosis, which affect the normal activity of cardiomyocytes. Meanwhile, the fission and fusion of mitochondria is in a delicate balance under normal circumstances. DRP1 is a GTPase protein which is a key player in fission. Under a variety of stresses, including hypoxia and oxidative stress. The translocation of Drp1 is from the cytosol to mitochondria outer membrane, phosphorylate Drp1 at serine 616 can activate mitochondrial fission [32]. Through fission, the damaged mitochondria are separated from healthy mitochondria. Moreover, the kinetics-related GTPases MFN2 and OPA1 control mitochondrial membrane fusion. Mitochondria fuse together via increased MFN2 (outer membrane fusion) and the activation of OPA1 (inner membrane fusion). Mitochondrial dynamics are controlled by the balance of fission and fusion, maintaining the mitochondrial structure and function.

Moreover, normal mitochondrial biogenesis is critical for the proper mitochondrial function as well. PGC$1 \mathrm{a}$ is critical to the transcriptional control of mitochondrial biogenesis. It is a member of a family of nuclear regulators of transcription, and PGC-1 coactivators play important roles in driving mitochondrial biogenesis and function in the heart. It has been shown that loss of PGC-1 a accelerates cardiac dysfunction after stress overload, which is associated with impairment of mitochondrial biogenesis. Nuclear respiratory factor 1 (Nrf1) can regulate gene expression and TFAM can construct mitochondrial DNA transcription and replication. Nrf1 directly regulates the expression of transcription factor A mitochondrial (TFAM) genes to integrate nuclear control of mitochondrial genome transcription and replication activity. These molecules are essential for mitochondrial biogenesis [33, 34].

In previous studies, researches mainly shown that QSYQ could improve myocardial energy metabolism and inhibit ATP depletion in myocardial cells, as well improve the swelling morphology of damaged mitochondria and impaired myocardial mitochondrial structure [18]. However, further studies on

Page $10 / 18$ 
mitochondrial biogenesis and dynamical balance require further elucidation. In order to assess changes in mitochondrial biogenesis, we determined the expression of PGC-1a-a major regulator of mitochondrial biogenesis, TFAM and Nrf1. Proteins involved in mitochondrial fusion (MFN-2 and OPA1) and fission (DRP1 and p-DRP1(Ser616)) were measured to assess the changes in mitochondrial dynamics. After QSYQ treatment, PGC-1a, TFAM and Nrf1 expressions were increased. And the expression of $p$ DRP1(Ser616) was reduced, while DRP1 expression remains no changes. In addition, OPA1 and MFN2 expressions were increased by QSYQ administration. These all indicated that QSYQ could promote mitochondrial biogenesis and ameliorate the disturbance of mitochondrial dynamics.

\section{Conclusions}

This study demonstrated that QSYQ protected against myocardial ferroptosis induced by CAL via improving mitochondrial dynamical homeostasis and biogenesis. Our research indicated that these findings might provide novel insights into the pharmacological actions of QSYQ for the treatment of cardiovascular diseases.

\section{Declarations}

Funding This research was funded by the National Natural Science Foundation of China (No. 81973506).

Data Availability All data included in this study are available upon request from the corresponding author.

Author's Contribution Fang Li, Yuanyuan Zhang, Boyang Yu and Junping Kou designed the study; Lingling Wu, Zhaoyang Fan, Jincheng Liu, Zekun Cui, Zixuan Huang, and Qingfei Zhao conducted experimental studies; Lingling Wu and Zhaoyang Fan performed data analysis; Lingling Wu and Zhaoyang Fan edited the manuscript. All authors read and approved the final manuscript.

Ethics Statement All procedures were conducted in accordance with the National Institutes of Health Guidelines for the Care and Use of Laboratory Animals and were approved by the Animal Ethics Committee of China Pharmaceutical University. And this article does not contain any studies with human participants performed by any of the authors.

Conflict of interest The authors declare no conflict of interest.

\section{References}

1. Li M, Izpisua Belmonte JC. Mending a faltering heart. Circ Res. 2016;118(2):344-51.

2. Mascolo A, di Mauro G, Cappetta D, et al. Current and future therapeutic perspective in chronic heart failure. Pharmacol Res. 2022;175:106035.

3. Lu LQ, Tian J, Luo XJ, Peng J. Targeting the pathways of regulated necrosis: a potential strategy for alleviation of cardio-cerebrovascular injury. Cell Mol Life Sci. 2021;78(1):63-78. 
4. Tang D, Chen X, Kang R, Kroemer G. Ferroptosis: molecular mechanisms and health implications. Cell Res. 2021;31(2):107-125.

5. Kovacs A, Herwig M, Budde $H$, et al. Interventricular differences of signaling pathways-mediated regulation of cardiomyocyte function in response to high oxidative stress in the post-ischemic failing rat heart. Antioxidants (Basel). 2021;10(6):964.

6. Rosca MG, Hoppel CL. Mitochondria in heart failure. Cardiovasc Res. 2010;88(1):40-50.

7. Zhou B, Tian R. Mitochondrial dysfunction in pathophysiology of heart failure. J Clin Invest. 2018;128(9):3716-26.

8. Wang J, Li C, Cao Y, et al. Mechanism of QSYQ on anti-apoptosis mediated by different subtypes of cyclooxygenase in AMI induced heart failure rats. BMC Complement Altern Med. 2015;15:352.

9. Tahrir FG, Langford D, Amini S, Mohseni Ahooyi T, Khalili K. Mitochondrial quality control in cardiac cells: Mechanisms and role in cardiac cell injury and disease. J Cell Physiol. 2019;234(6):8122-33.

10. Jhun BS, J OU, Adaniya SM, Cypress MW, Yoon Y. Adrenergic regulation of Drp1-driven mitochondrial fission in cardiac physio-pathology. Antioxidants (Basel). 2018;7(12):195.

11. Qiu Z, Wei Y, Song Q, et al. The role of myocardial mitochondrial quality control in heart failure. Front Pharmacol. 2019;10:1404.

12. Marin-Garcia J, Akhmedov AT. Mitochondrial dynamics and cell death in heart failure. Heart Fail Rev. 2016;21(2):123-36.

13. Cao H, Wang P, Li N, et al. Practice of comparative effectiveness research to identify treatment characteristics of similar chinese patent medicine for angina pectoris. Evid Based Complement Alternat Med. 2017;2017:7062714.

14. Mao J, Zhang J, Lam CSP, et al. Qishen Yiqi dripping pills for chronic ischaemic heart failure: results of the CACT-IHF randomized clinical trial. ESC heart failure. 2020;7(6):3881-90.

15. Du H, Xiao G, Xue Z, et al. QiShenYiQi ameliorates salt-induced hypertensive nephropathy by balancing ADRA1D and SIK1 expression in Dahl salt-sensitive rats. Biomed Pharmacother. 2021;141:111941.

16. Han JY, Li Q, Pan CS, Sun K, Fan JY. Effects and mechanisms of QiShenYiQi pills and major ingredients on myocardial microcirculatory disturbance, cardiac injury and fibrosis induced by ischemia-reperfusion. Pharmacol Res. 2019;147:104386.

17. Ruan G, Ren H, Zhang C, et al. Cardioprotective effects of QiShenYiQi dripping pills on transverse aortic constriction-induced heart failure in mice. Front Physiol. 2018;9:324.

18. Chen JR, Wei J, Wang LY, et al. Cardioprotection against ischemia/reperfusion injury by QiShenYiQi Pill(R) via ameliorate of multiple mitochondrial dysfunctions. Drug Des Devel Ther. 2015;9:3051-66.

19. Cui YC, Yan L, Pan CS, et al. The contribution of different components in QiShenYiQi Pills (R) to its potential to modulate energy metabolism in protection of ischemic myocardial injury. Front Physiol. 2018;9:389. 
20. Chang M, Cheng L, Shen Y, et al. Qishenyiqi dripping pill improves ventricular remodeling and function in patients with chronic heart failure: A pooled analysis. Medicine (Baltimore). 2019;98(2):e13906.

21. Wang H, Li L, Qing X, Zhang S, Li S. Efficacy of Qishen Yiqi drop pill for chronic heart failure: An Updated Meta-Analysis of 85 Studies. Cardiovasc Ther. 2020;2020:8138764.

22. Lin SQ, Wei XH, Huang P, et al. QiShenYiQi Pills(R) prevent cardiac ischemia-reperfusion injury via energy modulation. Int J Cardiol. 2013;168(2):967-74.

23. Fang $X$, Wang $H$, Han $D$, et al. Ferroptosis as a target for protection against cardiomyopathy. Proc Natl Acad Sci U S A. 2019;116(7):2672-80.

24. Wu X, Li Y, Zhang S, Zhou X. Ferroptosis as a novel therapeutic target for cardiovascular disease. Theranostics. 2021;11(7):3052-59.

25. Xie Y, Hou W, Song X, et al. Ferroptosis: process and function. Cell Death Differ. 2016;23(3):369-79.

26. Tsutsui $H$, Kinugawa S, Matsushima S. Oxidative stress and heart failure. Am J Physiol Heart Circ Physiol. 2011;301(6):H2181-90.

27. Song $\mathrm{M}$, Chen $\mathrm{Y}$, Gong $\mathrm{G}$, et al. Super-suppression of mitochondrial reactive oxygen species signaling impairs compensatory autophagy in primary mitophagic cardiomyopathy. Circ Res. 2014;115(3):34853.

28. Ravingerova T, Kindernay L, Bartekova M, et al. The molecular mechanisms of iron metabolism and its role in cardiac dysfunction and cardioprotection. Int J Mol Sci. 2020;21(21):7889.

29. Dabkowski ER, Williamson CL, Hollander JM. Mitochondria-specific transgenic overexpression of phospholipid hydroperoxide glutathione peroxidase (GPX4) attenuates ischemia/reperfusionassociated cardiac dysfunction. Free Radic Biol Med. 2008;45(6):855-65.

30. Yang WS, Stockwell BR. Ferroptosis: Death by Lipid Peroxidation. Trends Cell Biol. 2016;26(3):16576.

31. Wang Y, Li C, Ouyang Y, et al. QSYQ attenuates oxidative stress and apoptosis induced heart remodeling rats through different subtypes of NADPH-oxidase. Evid Based Complement Alternat Med. 2013;2013:824960.

32. Jin JY, Wei XX, Zhi XL, Wang XH, Meng D. Drp1-dependent mitochondrial fission in cardiovascular disease. Acta Pharmacol Sin. 2021;42(5):655-64.

33. Dominy JE, Puigserver P. Mitochondrial biogenesis through activation of nuclear signaling proteins. Cold Spring Harb Perspect Biol. 2013;5(7):a015008.

34. Dorn. Mitochondrial biogenesis and dynamics in the developing and diseased heart. GENES \& DEVELOPMENT. 2015;29(19):1981-91.

\section{Tables}

Tables are available in the Supplementary File Section. 
Fig. 1

a.
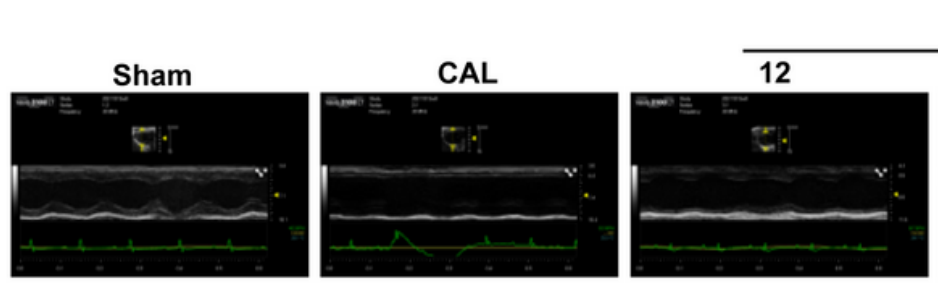

QSYQ(mg/kg)

b.

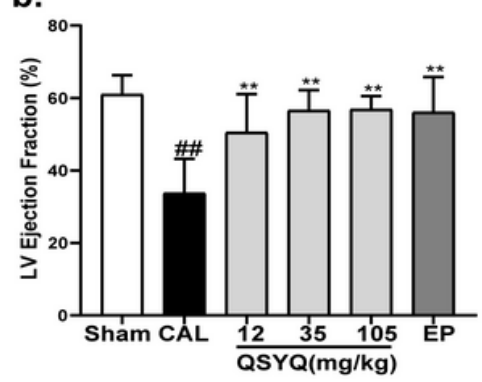

c.

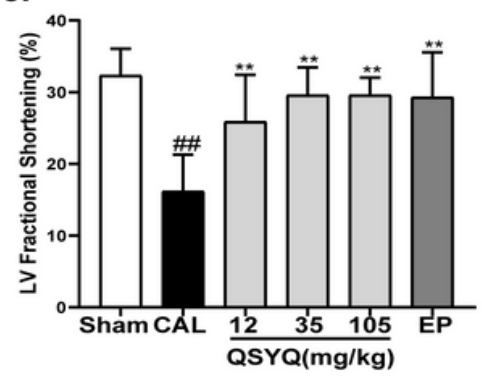

d.

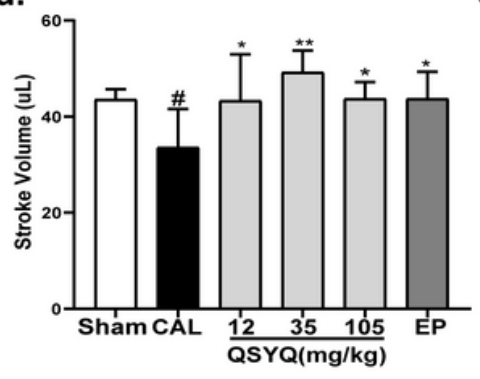

$E P(2.6 \mathrm{mg} / \mathrm{kg})$
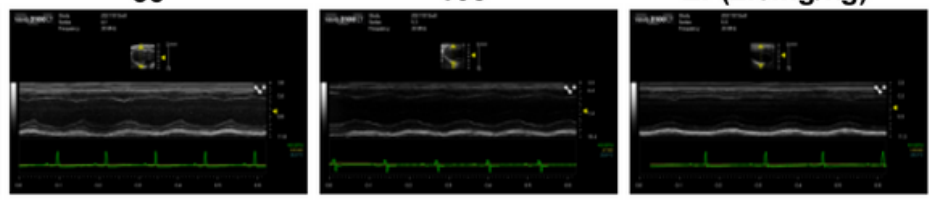

e.

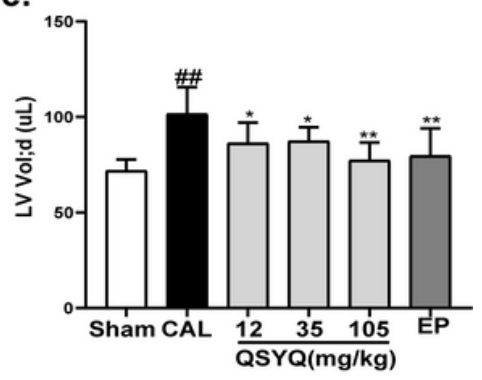

Figure 1

QSYQ effectively restored the cardiac contractile function in CAL-induced HF mice. (a) Representative images of M-mode echocardiograms. Effects of QSYQ on LVEF (b), LVFS (c), SV (d), LV vol (e) changes after 14 days of CAL in mice. Results were presented as mean \pm SD. ${ }^{\#} p<0.05,{ }^{\# \#} p<0.01$ vs. Sham group, ${ }^{*} p<0.05, * * p<0.01$ vs Model group. $\mathrm{n}=5$.

Fig. 2

a.
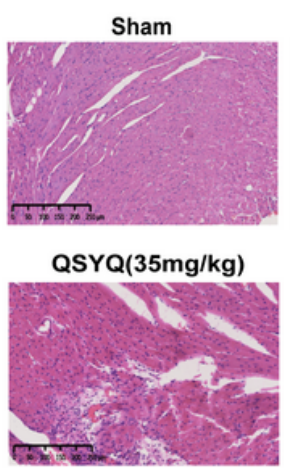

b.

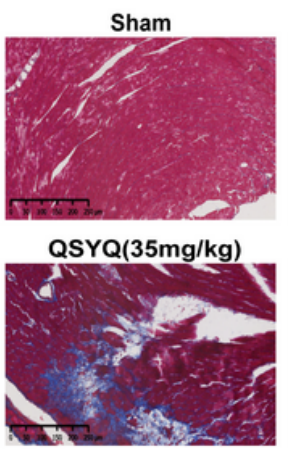

CAL

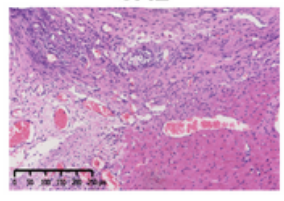

QSYQ(105mg/kg)

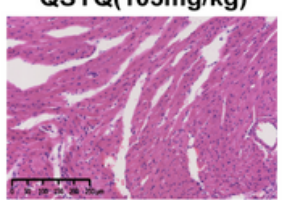

CAL

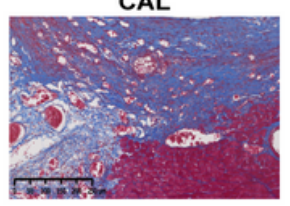

QSYQ(105mg/kg)
QSYQ(12mg/kg)

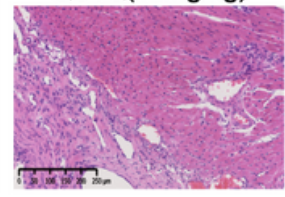

$E P(2.6 \mathrm{mg} / \mathrm{kg})$

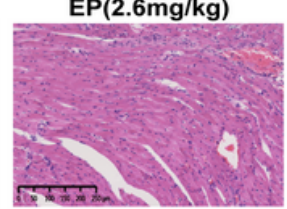

QSYQ(12mg/kg)

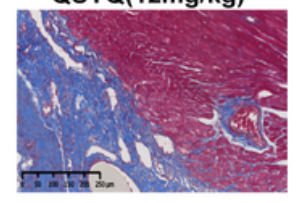

$E P(2.6 \mathrm{mg} / \mathrm{kg})$
C.

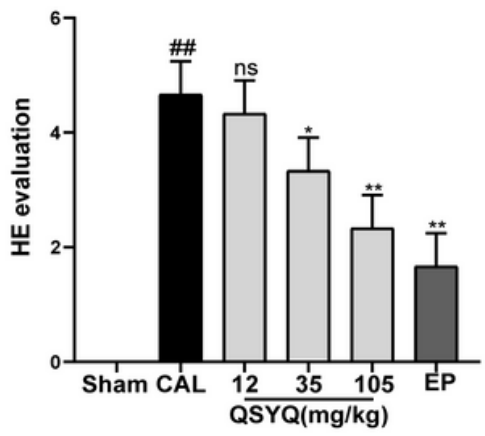

d.

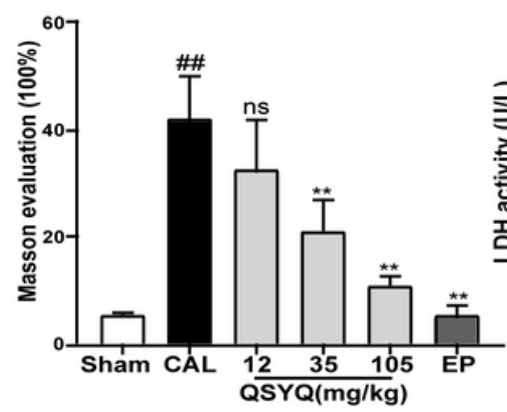

e.

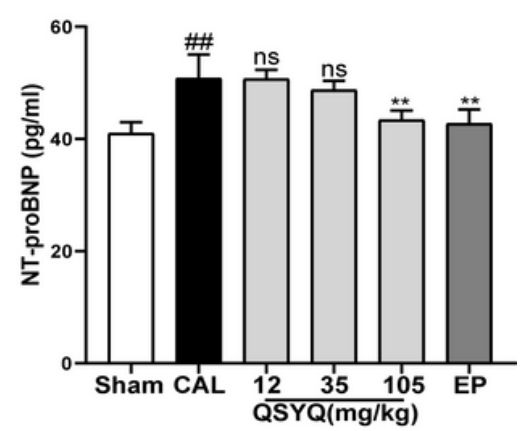

f.

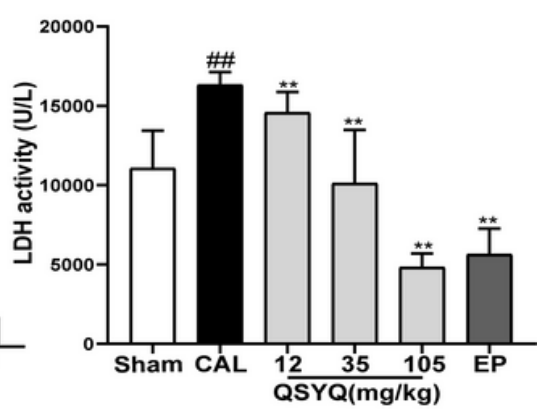


Figure 2

QSYQ attenuated myocardial injury in CAL-induced HF mice. (a, b) Representative HE and Masson staining results of heart tissue of mice. (c, d) Quantitative analysis results of HE and Masson staining. (e, f) NT-pro BNP and LDH activity of serum were examined. Results were presented as mean \pm SD. ${ }^{\#} p<0.05$, $\#$ \# $p<0.01$ vs. Sham group, ${ }^{*} p<0.05,{ }^{*} p<0.01$ vs Model group. $\mathrm{n}=3$.

Fig. 3

a.

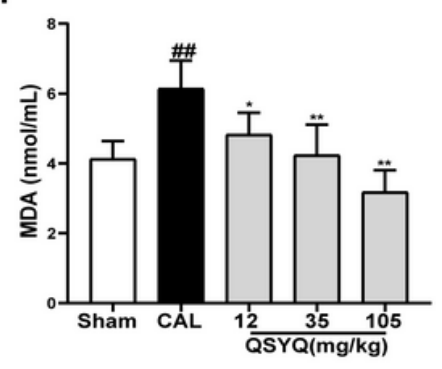

e.

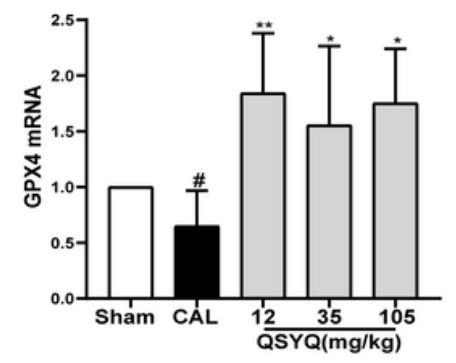

h.

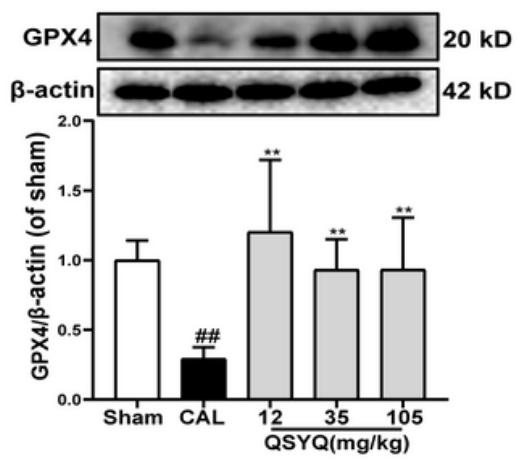

b.

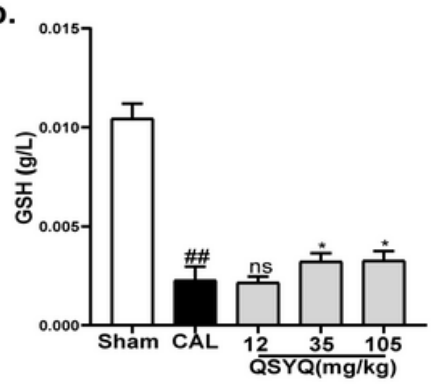

f.

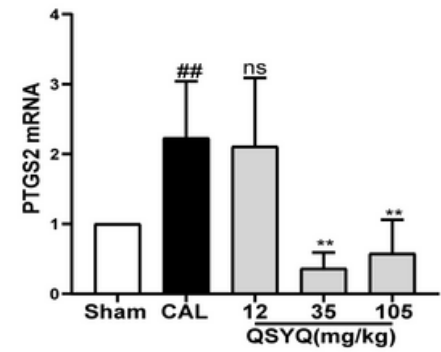

i.

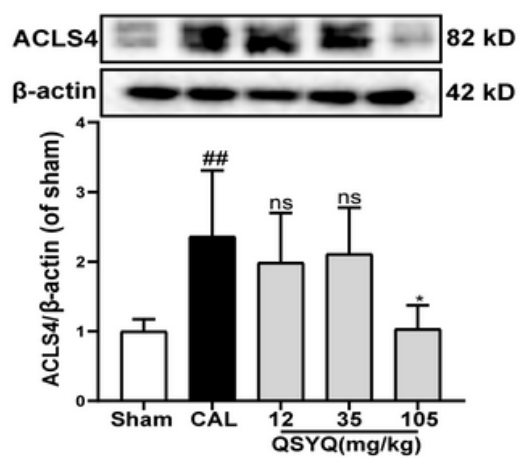

d.
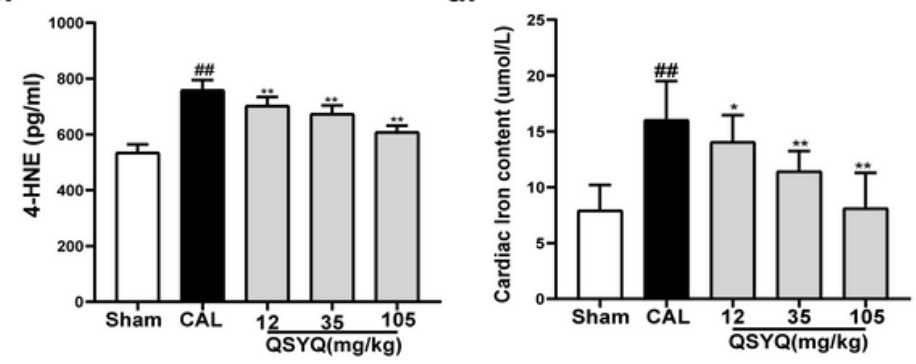

g.

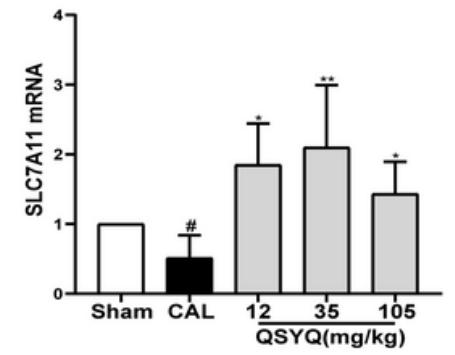

j.

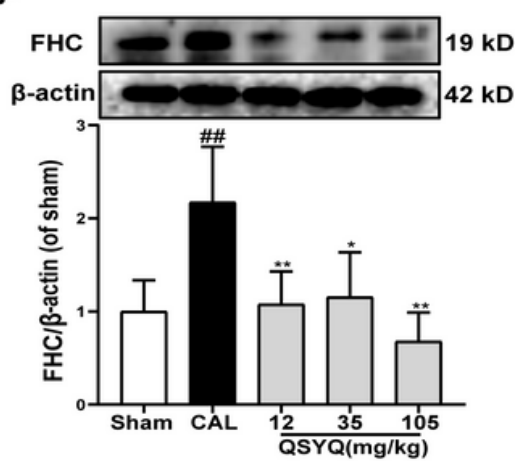

Figure 3

QSYQ mitigated ferroptosis in CAL-Induced HF mice. (a-d) The levels of MDA, GSH, 4-HNE and total iron were assayed. (e-g) RT-PCR results of GPX4, SLC7A11 and PTGS2. (h-j) Western blotting results of GPX4, ACSL4 and FHC. The representative images were shown and results were presented as mean \pm SD. ${ }^{\#} p<$ $0.05,{ }^{\# \#} p<0.01$ vs. Sham group, ${ }^{\star} p<0.05,{ }^{\star \star} p<0.01$ vs Model group. $\mathrm{n}=5$. 
Fig. 4

a.
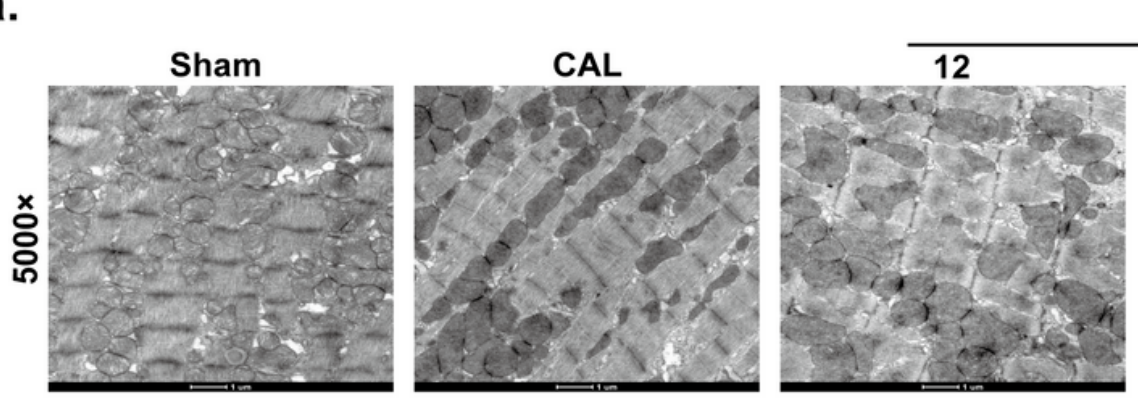

QSYQ(mg/kg)
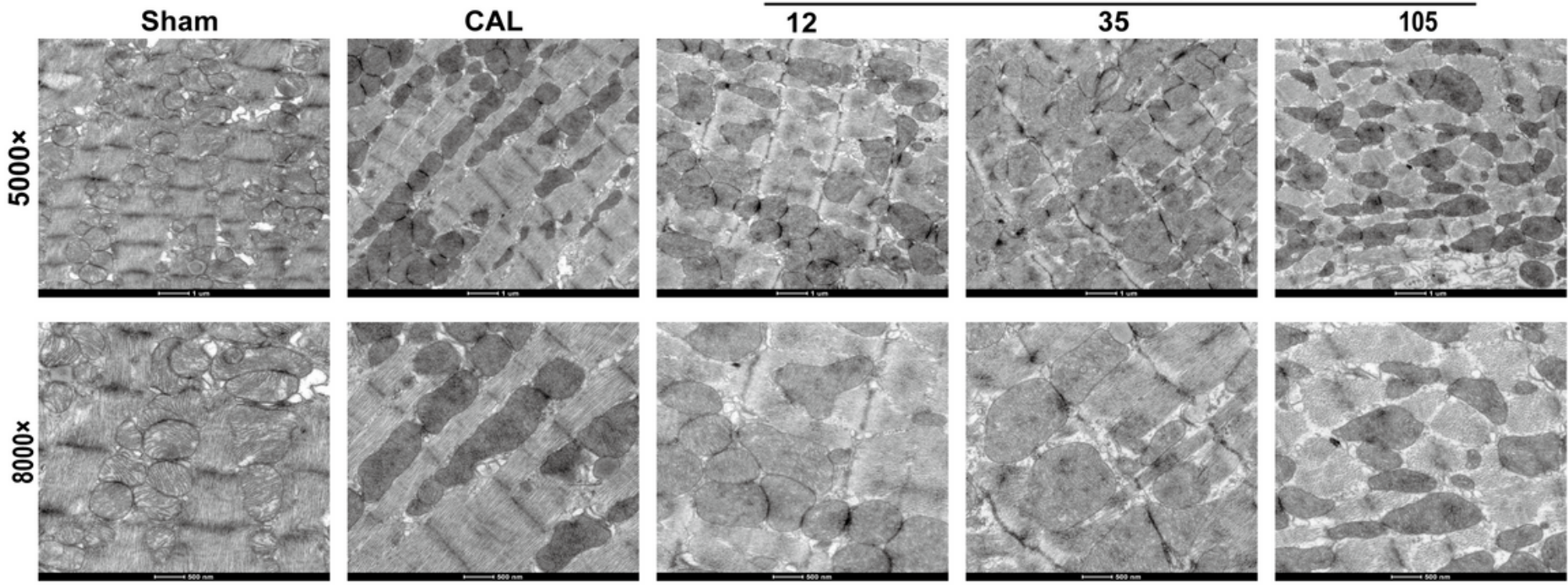

b.

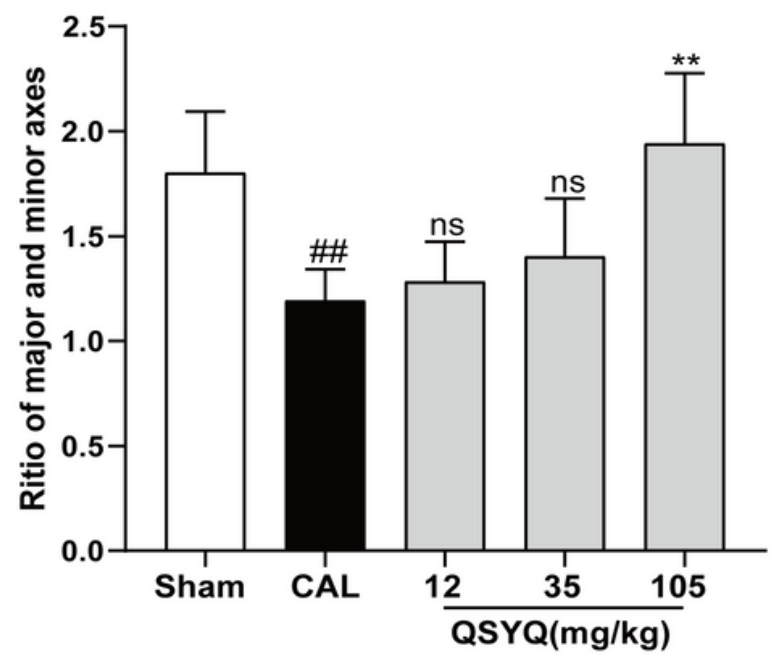

C.

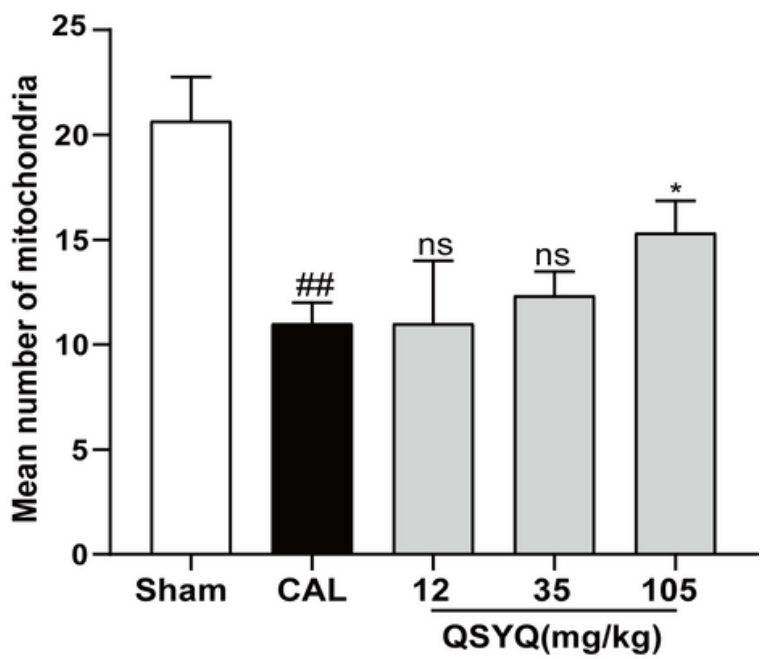

Figure 4

QSYQ improved the mitochondrial ultrastructure damage in CAL-induced HF mice. (a) Representative photomicrographs $(\times 5000,8000)$ of the mitochondrial morphology. (b) The mean number of mitochondria was measured. (c) The ratio of major and minor axes of mitochondria was calculated. Results were presented as mean \pm SD. ${ }^{\#} p<0.05,{ }^{\# \#} p<0.01$ vs. Sham group, ${ }^{\star} p<0.05,{ }^{\star \star} p<0.01$ vs Model group. $\mathrm{n}=3$. 


\section{Fig. 5}

a.

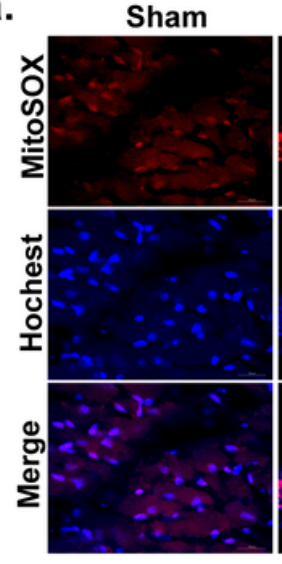

CAL

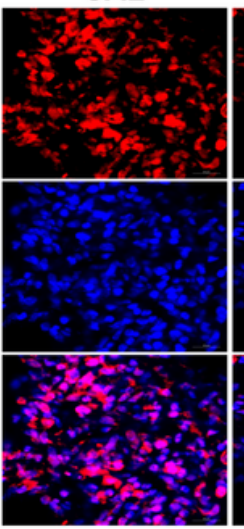

QSYQ $(\mathrm{mg} / \mathrm{kg})$

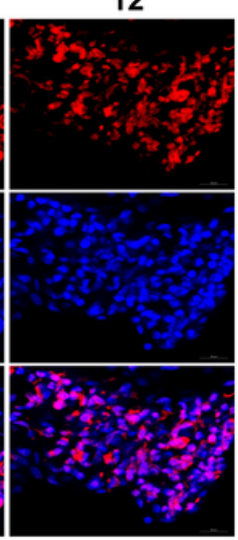

105

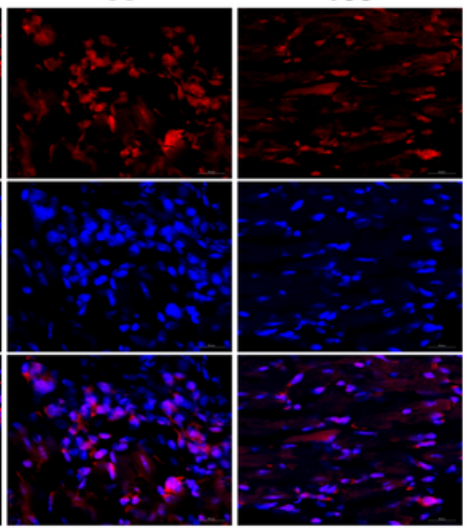

b.

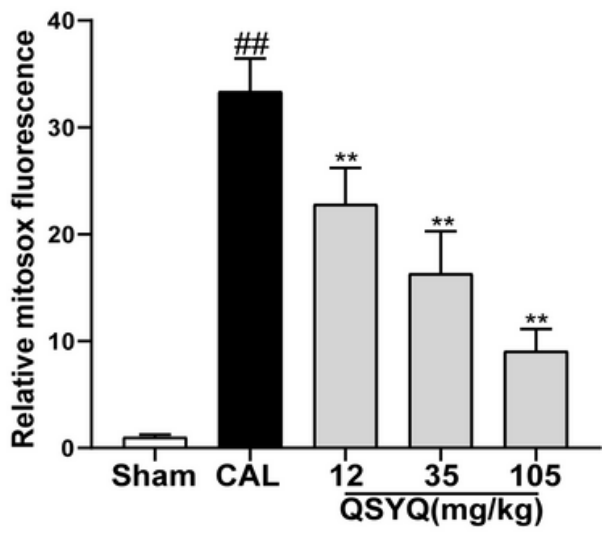

\section{Figure 5}

QSYQ decreased mitochondrial ROS production in CAL-induced HF Mice. (a) Representative images of the fluorescence. (b) Relative Mito-sox fluorescence were presented as mean \pm SD. ${ }^{\#} p<0.05$, ${ }^{\# \#} p<0.01$ vs. Sham group, ${ }^{\star} p<0.05,{ }^{*} p<0.01$ vs Model group. $\mathrm{n}=3$.

Fig. 6

a.

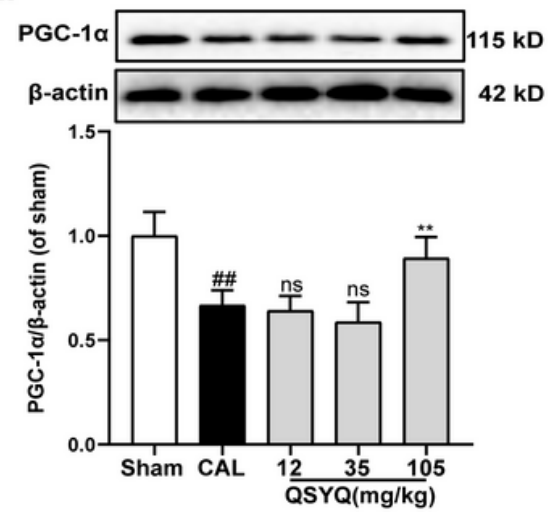

b.

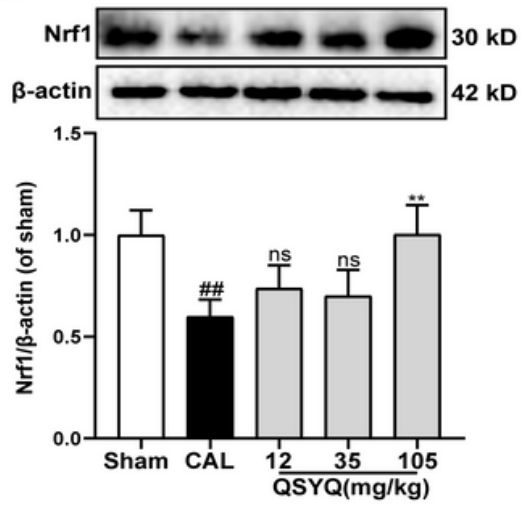

c.

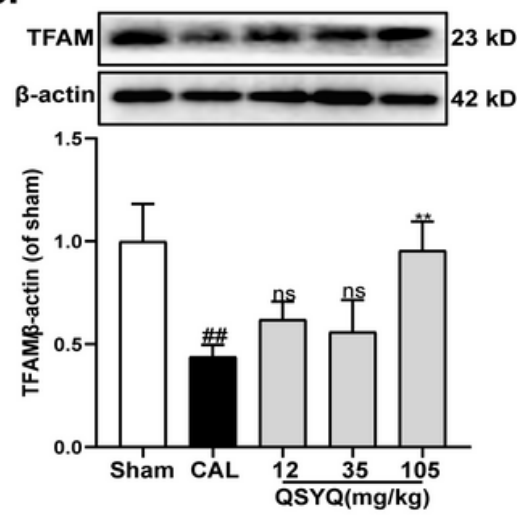

Figure 6

QSYQ promoted mitochondrial biogenesis in CAL-induced HF Mice. The PGC-1a(a), TFAM (b), Nrf1 (c) expression were detected by using Western blotting analysis. All data were shown as the mean \pm SD. ${ }^{\#} p<$ $0.05,{ }^{\# \#} p<0.01$ vs. Sham group, ${ }^{*} p<0.05,{ }^{\star *} p<0.01$ vs. Model group. $\mathrm{n}=5$. 
Fig. 7

a.
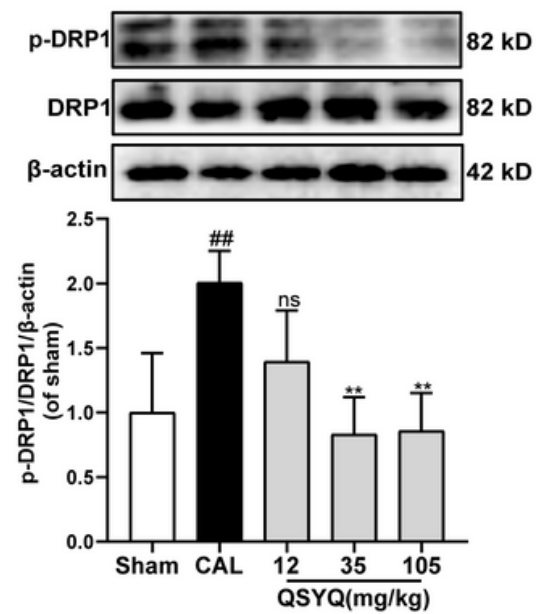

b.
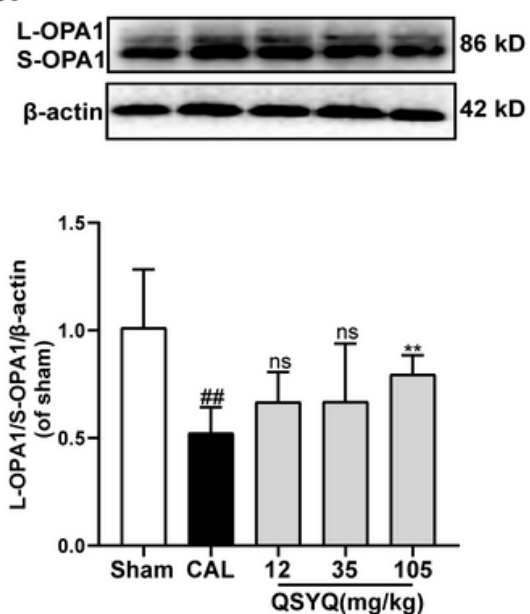

c.
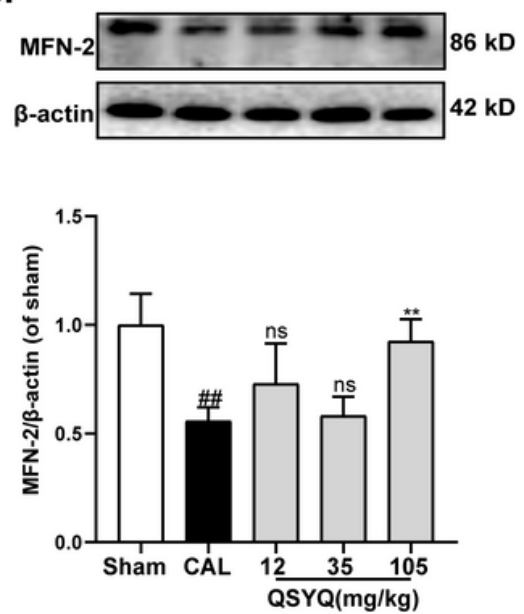

Figure 7

QSYQ improved mitochondrial dynamical homeostasis in CAL-induced HF mice. (a) p-DRP1, DRP1 (b) OPA1 (c) MFN-2 expression were detected using Western blotting analysis. All data are shown as the mean \pm SD. ${ }^{\#} p<0.05,{ }^{\#} p<0.01$ vs. Sham group, ${ }^{*} p<0.05,{ }^{* *} p<0.01$ vs. Model group. $\mathrm{n}=5$.

\section{Supplementary Files}

This is a list of supplementary files associated with this preprint. Click to download.

- Table1.xlsx

- GraphicalAbstract.png 